\title{
Effects of adiposity on the human plasma proteome: observational and Mendelian randomisation estimates
}

\author{
Lucy J. Goudswaard $\mathbb{D}^{1,2,3,4}$, Joshua A. Bell ${ }^{1,2}$, David A. Hughes $\mathbb{D}^{1,2}$, Laura J. Corbin (D) ${ }^{1,2}$, Klaudia Walter (D) ${ }^{5}$, George Davey Smith ${ }^{1,2}$, \\ Nicole Soranzo $5,6,7$, John Danesh $5,7,8,9,10,11$, Emanuele Di Angelantonio $7,8,9,10,11$, Willem H. Ouwehand (D) ${ }^{5,6,9,12}$, Nicholas A. Watkins ${ }^{12}$, \\ David J. Roberts ${ }^{7,13,14}$, Adam S. Butterworth (D) ${ }^{7,8,9,10,11}$, Ingeborg Hers ${ }^{3,4}$ and Nicholas J. Timpson (iD ${ }^{1,2}$
}

(c) The Author(s) 2021

BACKGROUND: Variation in adiposity is associated with cardiometabolic disease outcomes, but mechanisms leading from this exposure to disease are unclear. This study aimed to estimate effects of body mass index (BMI) on an extensive set of circulating proteins.

METHODS: We used SomaLogic proteomic data from up to 2737 healthy participants from the INTERVAL study. Associations between self-reported BMI and 3622 unique plasma proteins were explored using linear regression. These were complemented by Mendelian randomisation (MR) analyses using a genetic risk score (GRS) comprised of 654 BMl-associated polymorphisms from a recent genome-wide association study (GWAS) of adult BMI. A disease enrichment analysis was performed using DAVID Bioinformatics 6.8 for proteins which were altered by BMI.

RESULTS: Observationally, BMI was associated with 1576 proteins $\left(P<1.4 \times 10^{-5}\right)$, with particularly strong evidence for a positive association with leptin and fatty acid-binding protein-4 (FABP4), and a negative association with sex hormone-binding globulin (SHBG). Observational estimates were likely confounded, but the GRS for BMI did not associate with measured confounders. MR analyses provided evidence for a causal relationship between BMI and eight proteins including leptin (0.63 standard deviation (SD) per SD BMI, 95\% Cl 0.48-0.79, $\left.P=1.6 \times 10^{-15}\right)$, FABP4 (0.64 SD per SD BMl, 95\% Cl 0.46-0.83, $\left.P=6.7 \times 10^{-12}\right)$ and SHBG $(-0.45$ SD per SD BMI, $95 \% \mathrm{Cl}-0.65$ to $\left.-0.25, P=1.4 \times 10^{-5}\right)$. There was agreement in the magnitude of observational and MR estimates $\left(R^{2}\right.$ $=0.33$ ) and evidence that proteins most strongly altered by BMI were enriched for genes involved in cardiovascular disease. CONCLUSIONS: This study provides evidence for a broad impact of adiposity on the human proteome. Proteins strongly altered by $\mathrm{BMI}$ include those involved in regulating appetite, sex hormones and inflammation; such proteins are also enriched for cardiovascular disease-related genes. Altogether, results help focus attention onto new proteomic signatures of obesity-related disease.

International Journal of Obesity (2021) 45:2221-2229; https://doi.org/10.1038/s41366-021-00896-1

\section{INTRODUCTION}

Obesity has tripled worldwide since 1975 , now affecting around $40 \%$ of adults in the United States and $26 \%$ of adults in the UK [1]. The average body mass index (BMI) of the UK adult population is now in the conventional 'overweight' category (BMI between 25 and $30 \mathrm{~kg} /$ $\mathrm{m}^{2}$ ) [2] and 'overweight' is now more common than 'normal-weight' in middle age in many high-income countries [3]. BMI is often used as a proxy for adiposity given high correlations between BMI and more objectively measured fat mass indices [4]. Higher adiposity is a major risk factor for various noncommunicable diseases including type II diabetes, cardiovascular diseases, musculoskeletal diseases, and cancer [5-8], which collectively put a strain on health services $[9,10]$. These BMI-disease associations are supported by prospective observational studies and, more recently, by Mendelian randomisation (MR) studies [11-13], which use genetic variation reliably associated with BMI to re-estimate effects of BMI on disease outcomes. Given the properties of genetic variation, this method helps to overcome issues such as confounding and reverse causation which commonly occur with observational studies [14].

\footnotetext{
${ }^{1}$ Medical Research Council (MRC) Integrative Epidemiology Unit at the University of Bristol, Bristol, UK. ${ }^{2}$ Population Health Sciences, Bristol Medical School, University of Bristol, Bristol, UK. ${ }^{3}$ School of Physiology, Pharmacology and Neuroscience, University of Bristol, Bristol, UK. ${ }^{4}$ Bristol Heart Institute, Bristol, UK. ${ }^{5}$ Wellcome Sanger Institute, Hinxton, UK. ${ }^{6}$ Department of Haematology, School of Clinical Medicine, University of Cambridge, Cambridge, UK. ${ }^{7}$ NIHR Blood and Transplant Research Unit in Donor Health and Genomics, Department of Public Health and Primary Care, University of Cambridge, Cambridge, UK. ${ }^{8} \mathrm{MRC} / \mathrm{BHF}$ Cardiovascular Epidemiology Unit, Department of Public Health and Primary Care, University of Cambridge, Cambridge, UK. ${ }^{9}$ British Heart Foundation Centre of Research Excellence, University of Cambridge, Cambridge, UK. ${ }^{10}$ NIHR Cambridge Biomedical Research Centre, University of Cambridge and Cambridge University Hospitals, Cambridge, UK. ${ }^{11}$ Health Data Research UK Cambridge, Wellcome Genome Campus and University of Cambridge, Cambridge, UK. ${ }^{12} \mathrm{NHS}$ Blood and Transplant, Cambridge Biomedical Campus, Cambridge, UK. ${ }^{13} \mathrm{NHS}$ Blood and Transplant-Oxford Centre, Level 2, John Radcliffe Hospital, Oxford, UK. ${ }^{14}$ Radcliffe Department of Medicine, University of Oxford, John Radcliffe Hospital, Oxford, UK. ${ }^{凶}$ email: Ig14289@bristol.ac.uk
} 
Despite MR studies supporting a causal role of adiposity for cardiometabolic diseases, and randomised trials supporting the effectiveness of weight loss in reducing disease risk [15], the molecular footprint of adiposity is not well understood. Previous studies have largely focused on the impact of higher BMI on the lipidome including traits such as cholesterol and triglycerides in lipoprotein subtypes (e.g. low-density lipoprotein and highdensity lipoprotein particles) [4, 16], and on inflammatory molecules such as C-reactive protein (CRP) $[16,17]$.

A benefit of studying the systematic effects of BMI on the circulating proteome is that proteins are often more suitable pharmacological targets than metabolites. Efforts to study the effect of BMI on the proteome have generally been in an observational framework [18]. It is estimated that $25 \%$ of proteins in the human proteome circulate in blood [19], which is important as the majority of druggable targets are such proteins [20]. Studying the effect of $\mathrm{BMI}$ on a large set of proteins has only recently become possible with newly developed proteomic technologies such as the SomaLogic platform, with the ability to quantify enzymes, protein kinases and transport proteins with unprecedented sensitivity [21]. Utilisation of SomaLogic within a trial or cohort setting has recently become more widespread, such as within the INTERVAL study, a UK cohort of blood donors [22]. There is evidence that proteins which change as a result of a higher BMI may contribute to cardiometabolic disease [23]: identification of such proteins is important in understanding how higher BMI causes disease and to identify targets which may benefit from pharmacological intervention.

In this study, we aimed to measure associations between adiposity and the human proteome and to also estimate the underlying effects in a causal framework. Using data on 2737 participants from INTERVAL, we estimated effects of BMI on 4034 (3622 unique) plasma protein traits in both observational and MR frameworks. We examined the agreement between effect estimates from different methods and performed enrichment analyses of the most strongly altered proteins to map their potential relevance to disease.

\section{METHODS \\ Study population}

INTERVAL is a prospective cohort study, which was initially a randomised trial that aimed to test the efficiency and safety of reducing the time between whole blood donation in $\sim 50,000$ participants [24]. Upon informed consent, eligible participants who were: aged 18 years and over, willing to complete online questionnaires and without a self-reported history of major disease were recruited between June 11th, 2012 and June 15th, 2014 from 25 National Health Service Blood and Transplant (NHSBT) centres across England. Participants filled out questionnaires including selfreported height and weight, smoking frequency and alcohol consumption. Blood samples were taken at baseline which were analysed for full blood counts and blood biomarkers. This study was approved by Cambridge (East) Research Ethics Committee. Access to the data was granted by the Data Access Committee.

The present study was conducted on a random subset of participants from INTERVAL who had basic phenotype data and plasma proteins measured by SomaLogic. This included up to 2737 participants mostly of European descent across analyses described below.

\section{Assessment of BMI and covariables}

Participants completed online questionnaires wherein they reported their height and weight. BMI was calculated as weight in kilograms divided by the square of their height in metres $\left(\mathrm{kg} / \mathrm{m}^{2}\right)$. Available covariables were age, sex, previous or current smoking frequency (in three categories of: never, occasional, most days or every day) and alcohol intake frequency (in four categories of: rarely, less than once a week, 1-2 times a week, 3-5 times a week or most days). These covariables were chosen as they were measured in the INTERVAL collection and are measures which are thought to influence adiposity and cardiometabolic health [4].

\section{Measurement of circulating proteins}

Plasma proteins were measured in INTERVAL participants at baseline (before randomisation of assignment to the time interval between blood donation) using the SomaScan by SomaLogic [22]. This platform uses 4034 modified nucleotides known as Slow Off-rate Modified Aptamers (SOMAmers) which make direct contact with proteins, enabling detection of 3622 unique proteins or protein complexes and quantifies them using a DNA microarray [21]. Separate SOMAmers can bind to isoforms of the same protein, but can also bind to the same protein at different sites (which can be impacted by post-translational modifications or complexes formed with other proteins). We therefore have included all 4034 SOMAmers. The extensive number of proteins measured, with no missingness and in a cohort of 2737 participants, provides a rich proteomic dataset. The proteins were measured in relative fluorescence units and quality control (QC) was performed as described by Sun et al. [22]. There was no missingness across protein variables. The proteomic data used had been pre-adjusted (using linear regression) for age, sex, duration between blood draw and sample processing ( 1 day or less vs $>1$ day), and the first three genetic principal components, with the residuals inverse normal rank transformed. All following analyses use this "pre-adjusted" data as input.

\section{Genetic data and instrument for BMI}

INTERVAL participant genotyping was performed on the Affymetrix GeneTitan Multi-Channel (MC) Instrument using the UK Biobank Axiom ${ }^{\mathrm{m}}$ Array (ThermoFisher Scientific, Loughborough, UK) and the QC of genotype data was implemented as described by Astle et al. [25]. The imputation panel used was the 1000 genomes phase-3-UK-10K [25]. A genetic instrument for BMI was constructed using 654 genetic variants that were associated with $\mathrm{BMI}$ at $P<5 \times 10^{-8}$ in the inverse variance weighted fixed-effect meta-analysis of GWAS of $\sim 700,000$ individuals of European ancestry [26]. This meta-analysis consisted of $\sim 250,000$ adults from the Genetic Investigation of ANthromopetric Traits consortium [27] and $\sim 450,000$ adults from the UK Biobank study. Only $0.05 \%$ of UK Biobank participants were included in the current INTERVAL study (of $N=2737$ ). These participants were not excluded to increase power. The weighted GRS was made using PLINK 2.0 software [28] using the effect alleles and beta coefficients from the source GWAS. The score was calculated by multiplying the number of effect alleles at each SNP by its effect estimate (beta), summing these, and dividing by the total number of SNPs included. The GRS therefore can be interpreted as the average per-SNP effect on BMI for each individual.

\section{Statistical analyses}

The population characteristics of INTERVAL participants with SomaLogic data who were included in this study ( $N$ range: $2422-2737$ due to missing data for covariables) were compared to those INTERVAL participants who were not included ( $N$ range: $27,174-30,721$ ) to assess generalisability of any BMl-protein associations to the wider INTERVAL sample. Population characteristics evaluated were age, sex, weight, height, BMI, smoking frequency, and alcohol intake. Differences in population characteristics among the two INTERVAL sub-sets were tested by a two-sided $t$-test for continuous traits and a two-sided Chi-square test for categorical variables. Observational analyses were conducted using linear regression to examine associations between BMI (in normalised standard deviation (SD) units based on a rank normal transformation (rntransform() from "moosefun" package https://github.com/hughesevoanth/moosefun) and each standardised protein trait as a dependent (outcome) variable. Two linear models were used (using $I m$ () function from R "stats" package): (1) adjusted for age and sex and (2) additionally adjusted for smoking and alcohol consumption (each as an ordered categorical variable). Given that the procedure which generates the "pre-adjusted" data (adjustment for covariables before rank normal transformation of proteins) can reintroduce correlations [29], age and sex are again used as covariables here. The estimates derived from models (1) and (2) therefore reflect the normalised SD-unit difference in each protein trait per normalised SD-unit $\left(4.8 \mathrm{~kg} / \mathrm{m}^{2}\right)$ higher BMI. Associations of covariables with $\mathrm{BMI}$ and protein traits were also examined using linear regression.

A Shapiro-Wilk test was used to confirm whether the GRS showed a normal distribution. MR analyses were conducted using two-stage least squares (2SLS) regression models with robust standard errors (SE), using the systemfit function as part of the systemfit package [30], with measured $\mathrm{BMI}$ in SD units and the GRS for BMI as the instrumental variable. These MR estimates reflect the normalised SD-unit difference in each protein trait per normalised SD-unit $\left(4.8 \mathrm{~kg} / \mathrm{m}^{2}\right)$ higher BMI. We report estimates from the 
Table 1. Characteristics of included participants.

\begin{tabular}{|c|c|c|c|}
\hline \multicolumn{2}{|l|}{ Variable } & $\begin{array}{l}\text { Mean } \\
\text { (SD) or \% }\end{array}$ & $N$ \\
\hline \multicolumn{2}{|l|}{ Age (years) } & $45.0(14.1)$ & 2737 \\
\hline \multirow[t]{2}{*}{ Sex } & Male & $51.7 \%$ & 2737 \\
\hline & Female & $48.3 \%$ & \\
\hline \multicolumn{2}{|l|}{ Weight (kg) } & $78.5(16.1)$ & 2736 \\
\hline \multicolumn{2}{|l|}{ Height (cm) } & $172.7(9.7)$ & 2730 \\
\hline \multicolumn{2}{|c|}{ Body mass index $\left(\mathrm{kg} / \mathrm{m}^{2}\right)$} & $25.9(4.8)$ & 2729 \\
\hline \multirow{3}{*}{$\begin{array}{l}\text { Smoking } \\
\text { frequency }\end{array}$} & Never & $59.1 \%$ & 2675 \\
\hline & Occasional & $11.9 \%$ & \\
\hline & Most days or every day & $29.0 \%$ & \\
\hline \multirow{4}{*}{$\begin{array}{l}\text { Alcohol intake } \\
\text { frequency }\end{array}$} & Rarely & $11.7 \%$ & 2422 \\
\hline & Less than once a week & $16.9 \%$ & \\
\hline & $\begin{array}{l}\text { One or two } \\
\text { times a week }\end{array}$ & $37.4 \%$ & \\
\hline & $\begin{array}{l}\text { Three to five times a } \\
\text { week/most days }\end{array}$ & $34.1 \%$ & \\
\hline
\end{tabular}

direct linear associations between BMI and proteins as "observational" estimates and those from the 2SLS causal effect estimates as "MR estimates". Agreement between observational and MR estimates was examined using a separate linear regression. This was performed: (1) for all proteins and (2) excluding the proteins that fell below our $P$ value reference point for strong evidence (defined below) to examine whether agreement is limited to 'top hits' or applies throughout the effect distribution. Agreement between observational estimates and MR estimates would suggest that there are causal effects of BMI across the general proteome, with differences in estimates suggesting confounding of observational estimates.

To account for multiple testing, a Bonferroni correction was used to adjust results. This was informed by the correlation between proteins, adjusting only for the estimated number of independent traits (Supplementary Fig. 1). Correlation was assessed by a Spearman's correlation matrix. From a starting number of 4034, the number of independent proteins was 3655 (using a correlation cut-off of $r=0.8$ or tree cut height $=0.2$ between proteins, Supplementary Fig. 2 , dendrogram made using "iPVs" package https://github.com/hughesevoanth/iPVs). We utilised a Bonferroni adjusted $P$ value of $0.05 / 3655=1.4 \times 10^{-5}$ to indicate strong evidence in this sample. Full results are presented in the supplementary material.

\section{Enrichment analysis}

To investigate whether any protein groups showed a particularly strong relationship with $\mathrm{BMI}$ and disease (signal detection), protein features were clustered for further analysis. First, a principal component analysis (PCA; prcomp() function from the R "stats" package) on the proteins, not the individuals, was performed on the "pre-adjusted" (see above) dataset (Supplementary Fig. 3A-D). The top ' $n$ ' PC eigenvectors, as identified by a scree plot of the PCA eigenvalues (Supplementary Fig. 3E), were carried forward into an unsupervised k-means analysis (kmeans() function from the $\mathrm{R}$ "stats" package). Nineteen k-means analyses were run altering the value of $k$ (number of clusters) from 2 to 20 . To identify an appropriate number of protein clusters ( $k$ ) we generated a scree like plot (Supplementary Fig. 3F). Here we plotted the variance explained by clusters, for each $k$, as estimated as the sum of squares explained by clusters (betweenness) over the total sums of squares, and looked for the smallest $\mathrm{k}$ with the maximum variance explained (a plateau). In summary, we used a data reduction method (PCA) to identify major axes (PCs) of the protein data that were then utilised in a machine learning clustering algorithm (k-means) to identify clusters of proteins that share abundance similarities across individuals.

To explore whether there was a systematic difference in the association of proteins within these clusters and BMI, the beta coefficients from the observational linear regressions or MR models were transformed into their absolute values and divided by their SE. The absolute betas divided by their SEs in each cluster was compared using a one-tailed pairwise Wilcox test to identify which clusters showed a stronger association with BMI. For the cluster(s) showing evidence for larger absolute beta coefficients, an enrichment analysis was performed using DAVID bioinformatics resources 6.8 [31]. Enrichment was assessed by using the uniprot IDs for the proteins in the cluster and comparing these proteins with the uniprot IDs of the full SomaLogic protein list. Enrichment for protein involvement in disease (using the genetic association database disease classes [32]) of the protein cluster was assessed by fold enrichment and a Bonferroni-corrected $P$ value to account for multiple testing. Proteins that were associated with BMI in confounder-adjusted observational analyses at $P<1.4 \times 10^{-5}$ were also entered into the disease enrichment tool and compared with the total proteins (as described for cluster enrichment). Analyses were performed using $\mathrm{R}$ version 3.4.2 [33]. $\mathrm{R}$ code used for analyses is available upon request.

\section{RESULTS}

\section{Participant characteristics}

INTERVAL participants included in this study (those with proteomic data), had a mean age of 45.0 years (SD of 14.1 years) and $48.3 \%$ were female (Table 1). Mean BMI was $25.9 \mathrm{~kg} / \mathrm{m}^{2}$ (SD of $4.8 \mathrm{~kg} / \mathrm{m}^{2}$ ) and the majority of participants were non-smokers (59.1\%). Nearly a quarter (23.5\%) reported currently or previously smoking daily and $71.5 \%$ reported drinking alcohol at least once a week. Participants with proteomic data were representative of the full INTERVAL cohort (Supplementary Table 1).

\section{Observational estimates of associations of BMI with protein traits}

In a linear regression model adjusted for age and sex among 2729 adults, BMI (per SD higher) was associated with 1576 proteins (39\%) at the level $P<1.4 \times 10^{-5}$ (multiple testing reference point, Supplementary Table 2 ). In a second model additionally adjusting for frequencies of smoking and alcohol intake among 2380 adults, there were 1447 associations at the same reference point (Supplementary Table 3). The strongest positive associations were with leptin (0.74 SD, 95\% Cl $\left.0.71-0.77, P=9.9 \times 10^{-324}\right)$ and adipocyte fatty acid binding protein (FABP4) $(0.58 \mathrm{SD}, 95 \% \mathrm{Cl}$ $0.55-0.62, P=6.4 \times 10^{-211}$ ). BMI (per SD) was also strongly positively associated with inflammatory proteins such as Complement Factor I (0.46 SD, 95\% Cl 0.43-0.50, $\left.P=5.6 \times 10^{-122}\right)$ and CRP (0.44 SD, 95\% Cl $0.41-0.48, P=8.2 \times 10^{-112}$ ). BMI (per SD) also showed strong negative associations with proteins such as insulinlike growth factor-binding protein 2 (IGFBP2) $(-0.48$ SD, $95 \% \mathrm{Cl}$ -0.51 to $-0.44, P=2.7 \times 10^{-133}$ ) and sex hormone-binding globulin (SHBG) $(-0.43 \mathrm{SD}, 95 \% \mathrm{Cl}-0.47$ to $-0.39, P=2.4 \times$ $\left.10^{-106}\right)$.

\section{Observational associations of covariables with BMI and protein traits}

Age, sex and frequencies of smoking and alcohol intake were each associated with BMI (Supplementary Table 4). Males had a higher BMI than females $\left(0.17 \mathrm{SD}, 95 \% \mathrm{Cl} 0.10-0.25, P=5.8 \times 10^{-6}\right)$. Age was positively associated with BMI (0.01 SD higher per year older, $\left.95 \% \mathrm{Cl} 0.009-0.015, P=1.2 \times 10^{-18}\right)$. Smoking frequency was positively associated with BMI, but alcohol intake frequency was negatively associated with BMI. Covariables (age, sex, smoking and alcohol) showed associations with protein traits (Supplementary Tables 5-8 and Supplementary Fig. 4A-D). There was evidence for 18 associations between age and proteins, 26 associations between sex and proteins, 38 proteins associated with smoking and 137 proteins associated with alcohol at the Bonferroniadjusted level of $p<1.4 \times 10^{-5}$.

\section{Associations of the GRS for BMI with measured BMI and covariables}

The distribution of the GRS among participants was normal (mean $=0.08, \mathrm{SD}=0.29, W=0.99, P=0.73, N=2729)$. The GRS was associated with BMI, explaining $2.8 \%$ of its variance $\left(R^{2}=0.028\right.$, $P=1.6 \times 10^{-18}$, Table 2). There was no strong evidence of 
association between GRS and age $\left(R^{2}=0.001, P=0.11\right)$, sex $\left(R^{2}=\right.$ $\left.6 \times 10^{-5}, P=0.28\right)$, smoking frequency $\left(R^{2} \leq 0.0001, P=0.91\right)$, or alcohol intake $\left(R^{2}<0.0001, P=0.44\right)$.

MR estimates of associations between BMI and protein traits In MR analyses, eight unique BMI-protein associations were detected at the level $P<1.4 \times 10^{-5}$ (multiple testing reference point, Fig. 1). MR estimates provide an estimate of the causal association between protein (in SDs) per SD higher BMI. The strongest association of BMI was again with leptin (0.63 SD, 95\% $\left.\mathrm{Cl}=0.48-0.79 ; P=1.6 \times 10^{-15}\right)$; this was followed by the association with FABP4 $\left(0.65 \mathrm{SD}, 95 \% \mathrm{Cl}=0.46-0.83 ; P=6.7 \times 10^{-12}\right)$. A strong negative association was also seen between BMI (per SD) and SHBG $\left(-0.45 \mathrm{SD}, 95 \% \mathrm{Cl}-0.65\right.$ to $\left.-0.25, P=1.4 \times 10^{-5}\right)$. Other BMI-protein associations $\left(P<1.4 \times 10^{-5}\right)$ included positive associations with fumarylacetoacetase, inhibin $\beta C$ chain and complement $\mathrm{C} 5$, and negative associations with receptor-type tyrosine-protein phosphatase delta and PILR alpha-associated neural protein. Supplementary Table 9 provides the full MR results.

\section{Comparison of observational and MR estimates}

The distribution of $P$ values for associations between BMI and protein traits suggested an overrepresentation of signal for the observational estimates of BMI and protein traits; far more than expected from chance alone (Supplementary Fig. 5A). In contrast to this, the extent of this overrepresentation was reduced considerably in the MR (Supplementary Fig. 5B).

The unadjusted and confounder-adjusted regression coefficients for $\mathrm{BMI}$ and protein traits were strongly associated $(\beta=0.99$ SDs, $R^{2}=0.99, P=9.9 \times 10^{-324}$, Fig. $\left.2 A\right)$. Compared with the observational estimates, the MR estimates were less precise, but there was a strong positive association between the beta coefficients from observational and MR estimates $(\beta=0.68$ SDs, $R^{2}=0.33, P=9.9 \times 10^{-324}$, Fig. $2 \mathrm{~B}$ ). After removing the proteins where $P<1.4 \times 10^{-5}$, the strength of association between unadjusted and adjusted observational estimates remained, but the association between observational and MR estimates attenuated slightly (Supplementary Fig. 6A/B). These results suggest causal effects of BMI across the general proteome.

\section{Enrichment analysis of strongest BMI-protein associations}

In examining the clustering of proteins, visual representation using a scree plot suggested there were five PCs that explained $30.3 \%$ of the variance (Supplementary Fig. 3). After PC5 there was clear drop in variance explained, therefore other PCs were excluded. These five PCs were entered into a k-means analysis, which provided evidence for five clusters (grouping of individual proteins is included in Supplementary Table 9). To identify which cluster was most strongly affected by BMI, the median absolute beta coefficient divided by the SE for each cluster was compared with the overall estimate. Six of the proteins out of the eight strongest BMI-protein MR estimates were in cluster 2 (Supplementary Table 9). There was consistent evidence that cluster 2 showed a stronger association with $\mathrm{BMI}$ than the overall average BMI-protein effect both observationally (3.79 (IQR 1.62-7.06) vs 3.35 (IQR 1.57-5.83) respectively, $P=3.7 \times 10^{-4}$ ) and in MR $(0.85$ (IQR $0.41-1.46)$ vs 0.74 (IQR $0.32-1.14$ ), $P=5.3 \times 10^{-6}$, Supplementary Table 10). Cluster 2 showed consistent evidence of a having the largest BMI effect. Compared with the full protein list in SomaLogic, the proteins in cluster 2 were enriched for disease (Table 3), including cardiovascular disease (1.14 fold enrichment, $\left.P=1.3 \times 10^{-4}\right)$, renal disease (1.22 fold enrichment, $P=1.0 \times$ $\left.10^{-3}\right)$, cancer (1.1 fold enrichment, $P=9.5 \times 10^{-3}$ ) and metabolic disease ( 1.08 fold enrichment, $P=4.2 \times 10^{-2}$ ). No other individual cluster showed enrichment for disease. Enrichment for disease was also explored by comparing the proteins which had an association with BMI $\left(P<1.4 \times 10^{-5}\right)$ in the confounder-adjusted regression model with the total protein list. Compared with the 


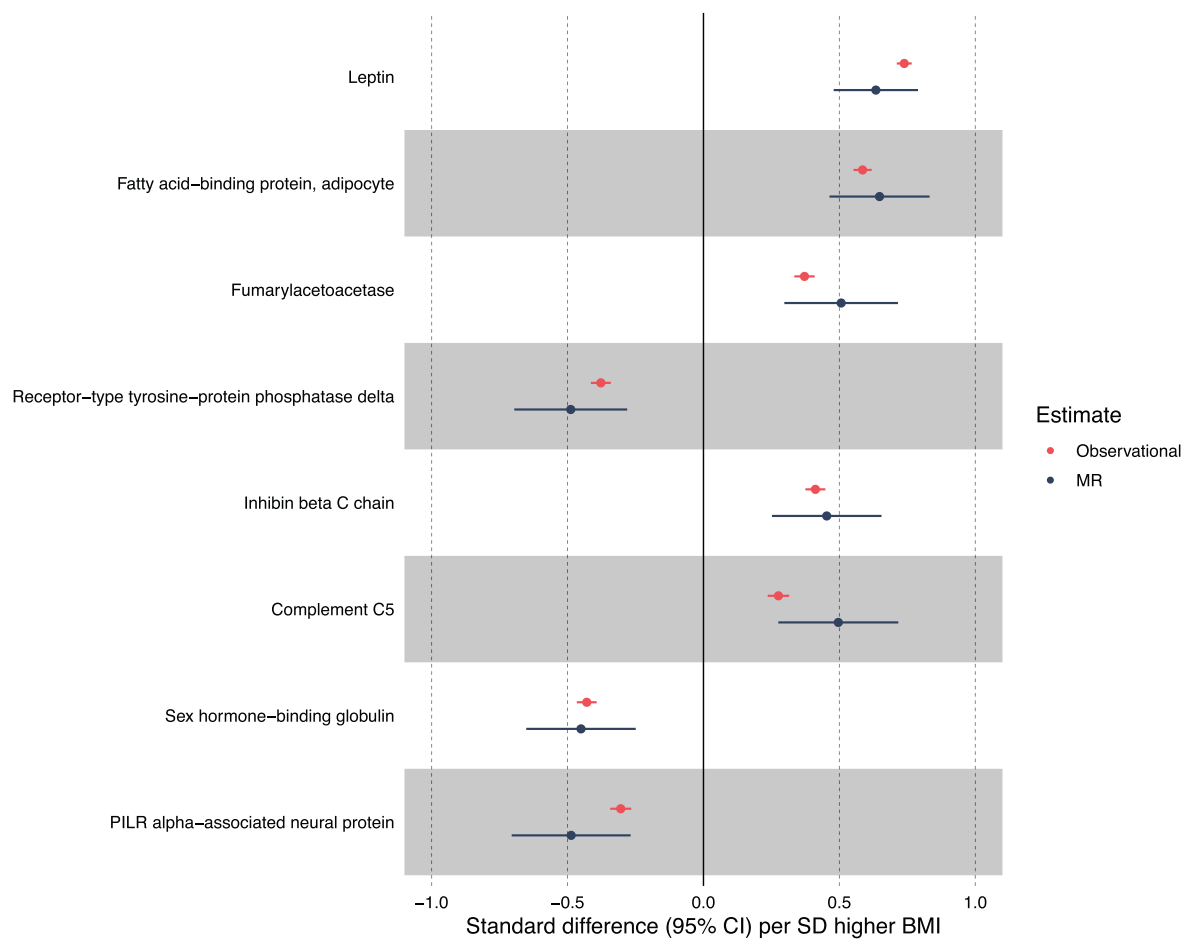

Fig. 1 Strongest BMI and protein Mendelian randomisation associations with corresponding observational associations. Forest plot of MR results of $\mathrm{BMI}$ and protein traits based on $P<1.4 \times 10^{-5}$ and their corresponding observational estimates.

full protein list, the proteins which showed a stronger observational association with $\mathrm{BMI}$ were enriched for renal disease (1.21 fold enrichment, $P=0.001)$ and metabolic disease $(1.9$ fold enrichment, $P=0.015$, Supplementary Table 11).

\section{DISCUSSION}

This study sought to estimate the effects of adiposity on a comprehensive set of protein traits only recently measurable by untargeted proteomics using observational and MR methods. Observational results provided evidence for associations between $\mathrm{BMI}$ and 1576 proteins, and MR was performed to reduce confounding. MR results suggest that BMI alters protein traits involved in regulating appetite, sex hormones, inflammation and other systems; specific proteins most altered by BMI include leptin, FABP4 and SHBG. Results of follow-up analyses suggest that the cluster of proteins most altered by $\mathrm{BMI}$ is enriched for genes associated with cardiovascular and metabolic disease.

This study explored the effect of BMI on a large set of circulating proteins in an MR framework. Previous studies have used observational epidemiology to explore the effect of obesity on the plasma proteome: one study used mass spectrometry and found an increase in Complement Factors $\mathrm{I}, \mathrm{B}$ and $\mathrm{H}$ and an increase in CRP [18]. These findings were replicated in our current observational analysis using the SomaLogic platform, indicating that associations are detectable across different proteomic platforms. The only association that did not replicate in the current study was the positive association with protein S100-A9. Although the MR analysis did not support some of these BMl-protein associations as being causal based on a $P$ value reference point, the strong association between the observational and MR estimates throughout the entire effect distribution suggests that disagreements between methods are likely an issue of power given current sample sizes.

Previous work implementing MR to examine the relationship between BMI and 1000 proteins (measured using the same SomaLogic array) provided corroborative evidence to that shown here [34]. Both studies suggested a positive association between $\mathrm{BMI}$ and leptin, as well as a negative association with SHBG. Other proteins, such as IGFBP1/2 and growth hormone receptor, did not pass our multiple testing threshold, but the direction and magnitude of estimates were in agreement, suggesting a possible causal effect that was not detectable in the current study. Building on previous work, the current study provides MR estimates for $>3600$ proteins, offering a wider proteomic profile and detecting additional associations such as that between BMI and fumarylacetoacetase and inhibin $\beta C$ chain. Furthermore, the inclusion of over threefold more proteins allowed a more comprehensive enrichment analysis to be performed.

For proteins with stronger MR-derived association evidence, it is important to explore whether they have a potential role in disease. Identification of individual proteins could help to guide future intervention if changes in proteins can be mapped to disease outcomes. Our results suggest a strong positive effect of BMI on levels of leptin, a hormone released by white adipose tissue which suppresses appetite [35]. The direction of effect agrees with estimates from previous cross-sectional and MR studies [16, 36], indicating leptin receptor resistance [37]. There is observational evidence in humans that higher leptin can induce greater aggregation of platelets (cells involved in haemostasis) [38]. In a larger observational study, leptin was found to be associated with higher risk of coronary events independent of BMI [39].

Our results help to provide contextualisation for proteins which have already been implicated in disease. For example, results suggest a strong positive effect of BMI on FABP4, an adipokine found primarily in adipocytes and macrophages [40]. This MR estimate supports the association which has been suggested in previous observational studies [41]. FABP4 has been implicated in cardiometabolic disease: a SNP which increases FABP4 was found to raise the odds of type II diabetes among adults [42], potentially through its contribution to higher insulin resistance [43]. FABP4 has also been associated with higher risk of atherosclerosis among adults [44]. A strong SHBG-lowering effect of higher BMI was also suggested here. The SHBG molecule is a glycoprotein which binds 
A

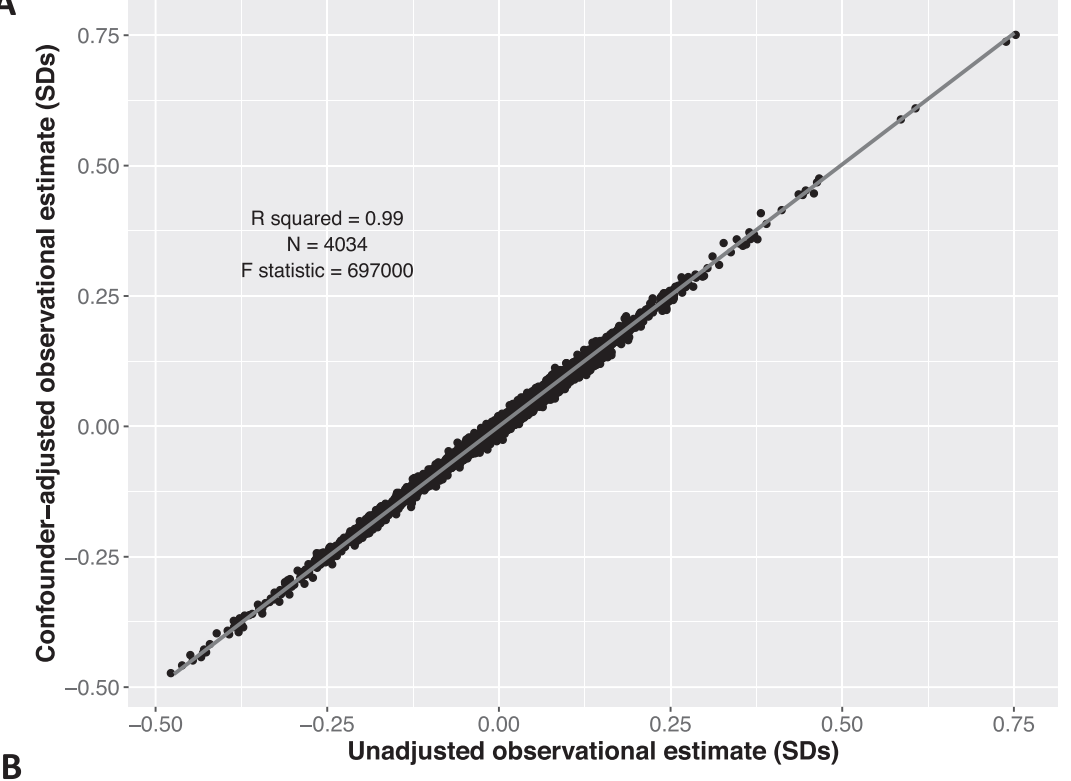

B

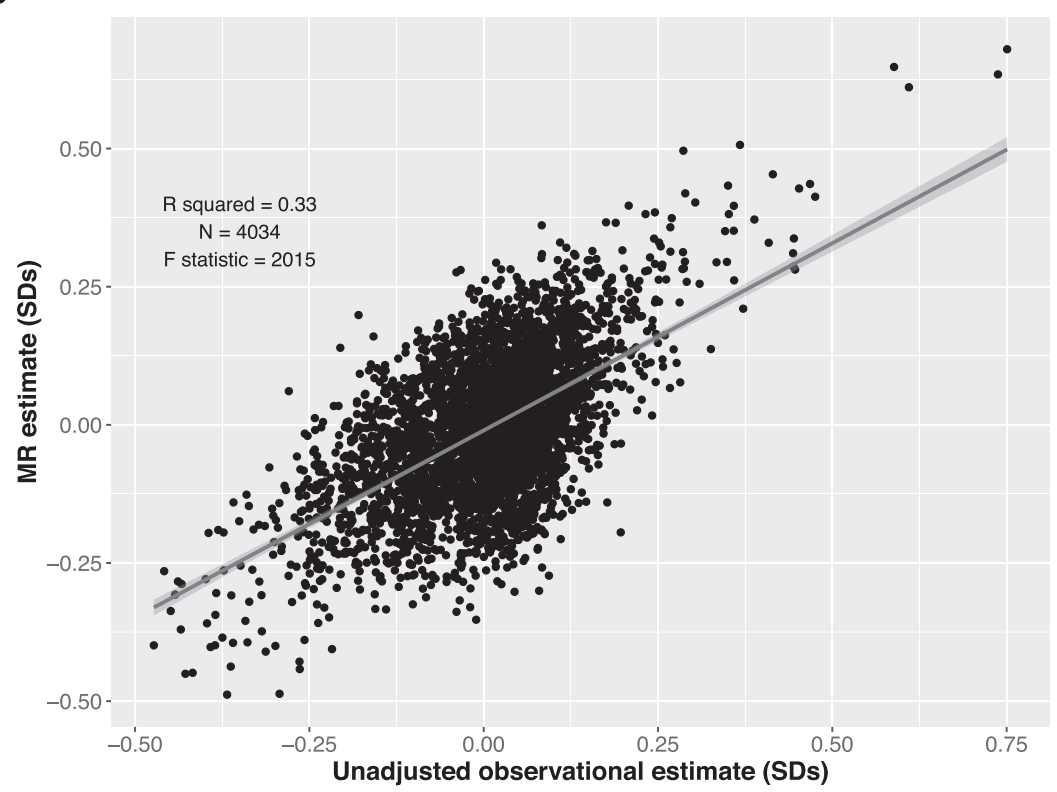

Fig. 2 Observational and Mendelian Randomisation estimates show a positive association. A Scatter plot of the unadjusted (age and sex adjusted) observational estimates and the confounder-adjusted observational estimates for BMI and protein traits with a regression line (blue). B Scatter plot of the unadjusted (age and sex adjusted) observational estimates and the MR estimates for BMI and protein traits with a regression line (blue).

androgens and oestrogens and suppresses their activity [45]; a reduction in SHBG is therefore expected to lead to higher levels of circulating sex hormones. The negative effect of BMI on SHBG seen here supports observational findings [46-48]. When evaluating the role of SHBG in disease, MR analysis suggests that an increase in SHBG contributes to a decrease in risk of cardioembolic stroke [49]. Other studies have also implicated lower SHBG levels in increasing type II diabetes risk $[42,50]$. The exact mechanisms leading from decreased SBHG to ill-health is unclear, but may arise as a result of the increased bioavailability of testosterone and oestrogen [45].

Despite these possible protein involvements in cardiometabolic disease, it remains difficult to assess the contribution of individual proteins as they are not entirely independent and any pathological effects would likely be due to a global change in protein composition. There are not distinct groupings in the SomaLogic data as there often are with, for example, metabolomics data. We therefore examined proteins grouped into clusters of similar features, compared BMI-protein estimates of each cluster with overall estimates and explored enrichment for genes related to disease. The cluster most altered by BMI (cluster 2) included most of the eight proteins with the strongest BMI effects from MR analyses, as well as various complement factors, chemokines and coagulation factors, and was found to be enriched for genes related to cardiovascular disease, renal and metabolic diseases and cancer. Enrichment was similar when comparing the proteins that had an observational association with BMI with all proteins included, with enrichment appearing greatest for renal and metabolic disease. Together, this suggests that changes in proteins may mediate effects of obesity on cardiometabolic diseases; more focused investigations of these proteins are now needed. 
Table 3. Cluster 2 vs full SomaLogic protein enrichment results for disease class using DAVID bioinformatics 6.8 [31].

\begin{tabular}{|c|c|c|c|c|c|c|c|c|}
\hline $\begin{array}{l}\text { Term (Genetic Association } \\
\text { Database disease class) }\end{array}$ & Count & $\%$ & List total & Population hits & Population total & $\begin{array}{l}\text { Fold } \\
\text { enrichment }\end{array}$ & $P$ value & $\begin{array}{l}\text { Bonferroni- } \\
\text { adjusted } \\
P \text { value }\end{array}$ \\
\hline Cardiovascular & 439 & 34.1 & 1024 & 1023 & 2723 & 1.14 & $7.3 \mathrm{E}-06$ & $1.3 \mathrm{E}-04$ \\
\hline Cancer & 401 & 31.2 & 1024 & 958 & 2723 & 1.11 & $5.3 \mathrm{E}-04$ & $9.5 \mathrm{E}-03$ \\
\hline Pharmacogenomic & 357 & 27.8 & 1024 & 856 & 2723 & 1.11 & $1.9 \mathrm{E}-03$ & $3.4 \mathrm{E}-02$ \\
\hline Haematological & 168 & 13.1 & 1024 & 388 & 2723 & 1.15 & $9.8 \mathrm{E}-03$ & $1.6 \mathrm{E}-01$ \\
\hline Reproduction & 152 & 11.8 & 1024 & 351 & 2723 & 1.15 & $1.4 \mathrm{E}-02$ & $2.3 \mathrm{E}-01$ \\
\hline Immune & 360 & 28.0 & 1024 & 886 & 2723 & 1.08 & $1.5 \mathrm{E}-02$ & $2.4 \mathrm{E}-01$ \\
\hline Neurological & 311 & 24.2 & 1024 & 759 & 2723 & 1.09 & $1.6 \mathrm{E}-02$ & $2.5 \mathrm{E}-01$ \\
\hline Aging & 117 & 9.1 & 1024 & 278 & 2723 & 1.12 & $7.5 \mathrm{E}-02$ & $7.5 \mathrm{E}-01$ \\
\hline
\end{tabular}

This study has some limitations. Firstly, although INTERVAL is one of the largest existing cohorts to have untargeted proteomic data based on the SomaLogic platform, the sample size is still relatively modest and may have low power to detect some associations when using MR: based on the detectable $(P<1.4 \times$ $10^{-5}$ ) median absolute observational effect size (0.13 SDs), our analyses had $80 \%$ power to detect MR effect sizes $\geq 0.33$ SDs $(a=$ $0.05)$ for our sample size $(N=2737)$ [51]. With greater statistical power, there would likely be more proteins detected with MR. This was reinforced by the high agreement in the magnitude of effect estimates seen in observational and MR analyses which applied throughout the effect distribution. Secondly, height and weight were self-reported which could bias results towards the null due to systematic error in BMI measurement. However, strong correlations are often reported between self-reported and measured BMI [52] and the validity of self-reported BMI is supported by the association between the GRS for BMI and selfreported BMI in INTERVAL to the degree expected. Thirdly, the small degree of overlap between INTERVAL and UK Biobank (participants used for the source GWAS for BMI who were also in INTERVAL) may have a biasing effect on estimates, though this is likely to be towards the null. We anticipate that overall, this bias would make estimates more conservative [53]. Fourth, we recognise a lack of availability of possible confounders such as socio-economic position (which likely affect both BMI and protein traits related to cardiovascular disease processes [54, 55]). Residual confounding may help account for the divergence between observed and expected $P$ values seen in observational vs MR models. Fifth, the proteins examined are highly correlated and we therefore may not fully be describing changes in individual proteins. Evidence from case-control cohorts as well as functional and animal studies would help isolate individual proteins that are altered and contribute to disease. Finally, although analyses provide insight into the proteomic effects of BMl, it does not distinguish between the type of adiposity. It would be useful to distinguish between the effects of subcutaneous and visceral fat using dual-energy X-ray absorptiometry derived measurements, but these were not available in the INTERVAL dataset.

This study utilised SomaLogic to explore the relationship between BMI and plasma proteins in unprecendented scope and detail, in both an observational and MR framework. We provide evidence for a broad impact of higher adiposity on the human proteome. Causal evidence was strongest for BMI in relation to proteins involved in regulating appetite, sex hormones and inflammation. Identification of BMI-driven protein changes could provide therapeutic targets for prevention of obesity-related disease. Protein alterations were also found to be enriched for genes related to cardiovascular and metabolic disease. Altogether, these results help to focus attention onto new potential proteomic signatures of obesity-related disease. Further characterisation of the role of such proteomic profiles in disease using MR is warranted.

\section{REFERENCES}

1. Blüher M. Obesity: global epidemiology and pathogenesis. Nat Rev Endocrinol. 2019;15:288-98.

2. Wade KH, Carslake D, Sattar N, Davey Smith G, Timpson NJ. BMI and Mortality in UK Biobank: revised Estimates Using Mendelian Randomization. Obesity (Silver Spring). 2018;26:1796-806.

3. (NCD2RisC) NRFC.Trends in adult body-mass index in 200 countries from 1975 to 2014: a pooled analysis of 1698 population-based measurement studies with 19.2 million participants. Lancet.2016;387:1377-96.

4. Bell JA, Carslake D, O'Keeffe LM, Frysz M, Howe LD, Hamer M, et al. Associations of Body Mass and Fat Indexes With Cardiometabolic Traits. J Am Coll Cardiol. 2018;72:3142-54.

5. Garg SK, Maurer H, Reed K, Selagamsetty R. Diabetes and cancer: two diseases with obesity as a common risk factor. Diabetes Obes Metab. 2014;16:97-110.

6. Khan SS, Ning H, Wilkins JT, Allen N, Carnethon M, Berry JD, et al. Association of Body Mass Index With Lifetime Risk of Cardiovascular Disease and Compression of Morbidity. JAMA Cardiol. 2018;3:280-7.

7. Kortt M, Baldry J. The association between musculoskeletal disorders and obesity. Aust Health Rev. 2002;25:207-14.

8. Bhaskaran K, Douglas I, Forbes H, dos-Santos-Silva I, Leon DA, Smeeth L. Bodymass index and risk of 22 specific cancers: a population-based cohort study of $5 \cdot 24$ million UK adults. Lancet. 2014;384:755-65.

9. Dixon P, Davey Smith G, Hollingworth W. The Association Between Adiposity and Inpatient Hospital Costs in the UK Biobank Cohort. Appl Health Econ Health Policy. 2019;17:359-70.

10. Dixon P, Hollingworth W, Harrison S, Davies NM, Davey Smith G. Mendelian Randomization analysis of the causal effect of adiposity on hospital costs. J Health Econ. 2020;70:102300.

11. Nordestgaard BG, Palmer TM, Benn M, Zacho J, Tybjaerg-Hansen A, Davey Smith G, et al. The effect of elevated body mass index on ischemic heart disease risk: causal estimates from a Mendelian randomisation approach. PLoS Med. 2012;9:e1001212.

12. Dale CE, Fatemifar G, Palmer TM, White J, Prieto-Merino D, Zabaneh D, et al. Causal Associations of Adiposity and Body Fat Distribution With Coronary Heart Disease, Stroke Subtypes, and Type 2 Diabetes Mellitus: a Mendelian Randomization Analysis. Circulation. 2017;135:2373-88.

13. Carreras-Torres R, Johansson M, Gaborieau V, Haycock PC, Wade KH, Relton CL, et al. The Role of Obesity, Type 2 Diabetes, and Metabolic Factors in Pancreatic Cancer: a Mendelian Randomization Study. J Natl Cancer Inst. 2017;109.

14. Smith GD, Ebrahim S. 'Mendelian randomization': can genetic epidemiology contribute to understanding environmental determinants of disease? Int J Epidemiol. 2003;32:1-22.

15. Wing RR, Lang W, Wadden TA, Safford M, Knowler WC, Bertoni AG, et al. Benefits of modest weight loss in improving cardiovascular risk factors in overweight and obese individuals with type 2 diabetes. Diabetes Care. 2011;34:1481-6.

16. Würtz P, Wang Q, Kangas AJ, Richmond RC, Skarp J, Tiainen M, et al. Metabolic signatures of adiposity in young adults: Mendelian randomization analysis and effects of weight change. PLoS Med. 2014;11:e1001765. 
17. Timpson NJ, Nordestgaard BG, Harbord RM, Zacho J, Frayling TM, TybjærgHansen A, et al. C-reactive protein levels and body mass index: elucidating direction of causation through reciprocal Mendelian randomization. Int J Obes (Lond). 2011;35:300-8.

18. Cominetti O, Núñez Galindo A, Corthésy J, Valsesia A, Irincheeva I, Kussmann M, et al. Obesity shows preserved plasma proteome in large independent clinical cohorts. Sci Rep. 2018;8:16981.

19. Gold L, Walker JJ, Wilcox SK, Williams S. Advances in human proteomics at high scale with the SOMAscan proteomics platform. N Biotechnol. 2012;29:543-9.

20. Imming $P$, Sinning $C$, Meyer A. Drugs, their targets and the nature and number of drug targets. Nat Rev Drug Discov. 2006;5:821-34.

21. Rohloff JC, Gelinas AD, Jarvis TC, Ochsner UA, Schneider DJ, Gold L, et al. Nucleic Acid Ligands With Protein-like Side Chains: modified Aptamers and Their Use as Diagnostic and Therapeutic Agents. Mol Ther Nucleic Acids. 2014;3:e201.

22. Sun BB, Maranville JC, Peters JE, Stacey D, Staley JR, Blackshaw J, et al. Genomic atlas of the human plasma proteome. Nature. 2018;558:73-9.

23. Garrido-Mesa N, Zarzuelo A, Gálvez J. Minocycline: far beyond an antibiotic. Br J Pharmacol. 2013;169:337-52.

24. Di Angelantonio E, Thompson SG, Kaptoge S, Moore C, Walker M, Armitage J, et al. Efficiency and safety of varying the frequency of whole blood donation (INTERVAL): a randomised trial of 45000 donors. Lancet. 2017;390:2360-71.

25. Astle WJ, Elding $H$, Jiang $T$, Allen $D$, Ruklisa $D$, Mann AL, et al. The Allelic Landscape of Human Blood Cell Trait Variation and Links to Common Complex Disease. Cell. 2016;167:1415-29.e19.

26. Yengo L, Sidorenko J, Kemper KE, Zheng Z, Wood AR, Weedon MN, et al. Metaanalysis of genome-wide association studies for height and body mass index in 700000 individuals of European ancestry. Hum Mol Genet. 2018;27:3641-9.

27. Locke AE, Kahali B, Berndt SI, Justice AE, Pers TH, Day FR, et al. Genetic studies of body mass index yield new insights for obesity biology. Nature. 2015;518: 197-206.

28. Purcell S, Neale B, Todd-Brown K, Thomas L, Ferreira MA, Bender D, et al. PLINK: a tool set for whole-genome association and population-based linkage analyses. Am J Hum Genet. 2007;81:559-75.

29. Pain O, Dudbridge F, Ronald A. Are your covariates under control? How normalization can re-introduce covariate effects. Eur J Hum Genet. 2018;26:1194-201.

30. Henningsen A, Hamann JD systemfit: a Package for Estimating Systems of Simultaneous Equations in R. 2007. 2007;23:40.

31. Huang dW, Sherman BT, Lempicki RA. Systematic and integrative analysis of large gene lists using DAVID bioinformatics resources. Nat Protoc. 2009;4:44-57.

32. Becker KG, Barnes KC, Bright TJ, Wang SA. The genetic association database. Nat Genet. 2004;36:431-2.

33. $R$ Core Team. $R$ : a language and environment for statistical computing. $R$ Foundation for Statistical Computing, Vienna, Austria. URL https://www.R-project.org/. 2019.

34. Zaghlool SB, Sharma S, Molnar M, Matías-García PR, Elhadad MA, Waldenberger $M$, et al. Revealing the role of the human blood plasma proteome in obesity using genetic drivers. Nat Commun. 2021;12:1279.

35. Klok MD, Jakobsdottir S, Drent ML. The role of leptin and ghrelin in the regulation of food intake and body weight in humans: a review. Obes Rev. 2007;8:21-34.

36. Millard LA, Davies NM, Timpson NJ, Tilling K, Flach PA, Davey Smith G. MRPheWAS: hypothesis prioritization among potential causal effects of body mass index on many outcomes, using Mendelian randomization. Sci Rep. 2015;5:16645.

37. Gruzdeva O, Borodkina D, Uchasova E, Dyleva Y, Barbarash O. Leptin resistance: underlying mechanisms and diagnosis. Diabetes Metab Syndr Obes. 2019;12:191-8.

38. Nakata M, Yada T, Soejima N, Maruyama I. Leptin promotes aggregation of human platelets via the long form of its receptor. Diabetes. 1999;48:426-9.

39. Wallace AM, McMahon AD, Packard CJ, Kelly A, Shepherd J, Gaw A, et al. Plasma leptin and the risk of cardiovascular disease in the west of Scotland coronary prevention study (WOSCOPS). Circulation. 2001;104:3052-6.

40. Furuhashi M, Saitoh S, Shimamoto K, Miura T. Fatty Acid-Binding Protein 4 (FABP4): pathophysiological Insights and Potent Clinical Biomarker of Metabolic and Cardiovascular Diseases. Clin Med Insights Cardiol. 2014;8 (Suppl 3):23-33.

41. Xu A, Wang Y, Xu JY, Stejskal D, Tam S, Zhang J, et al. Adipocyte fatty acidbinding protein is a plasma biomarker closely associated with obesity and metabolic syndrome. Clin Chem. 2006;52:405-13.

42. Gudmundsdottir V, Zaghlool SB, Emilsson V, Aspelund T, Ilkov M, Gudmundsson $\mathrm{EF}$, et al. Circulating protein signatures and causal candidates for type 2 diabetes. Diabetes. 2020;69:1843-53.

43. Nakamura R, Okura T, Fujioka Y, Sumi K, Matsuzawa K, Izawa S, et al. Serum fatty acid-binding protein 4 (FABP4) concentration is associated with insulin resistance in peripheral tissues, A clinical study. PLoS ONE. 2017;12:e0179737.

44. Yeung DC, Xu A, Cheung CW, Wat NM, Yau MH, Fong CH, et al. Serum adipocyte fatty acid-binding protein levels were independently associated with carotid atherosclerosis. Arterioscler Thromb Vasc Biol. 2007;27:1796-802.
45. Wallace IR, McKinley MC, Bell PM, Hunter SJ. Sex hormone binding globulin and insulin resistance. Clin Endocrinol (Oxf). 2013;78:321-9.

46. Cooper LA, Page ST, Amory JK, Anawalt BD, Matsumoto AM. The association of obesity with sex hormone-binding globulin is stronger than the association with ageing-implications for the interpretation of total testosterone measurements. Clin Endocrinol (Oxf). 2015;83:828-33.

47. Goto A, Chen BH, Song Y, Cauley J, Cummings SR, Farhat GN, et al. Age, body mass, usage of exogenous estrogen, and lifestyle factors in relation to circulating sex hormone-binding globulin concentrations in postmenopausal women. Clin Chem. 2014;60:174-85.

48. Baglietto L, English DR, Hopper JL, Maclnnis RJ, Morris HA, Tilley WD, et al. Circulating steroid hormone concentrations in postmenopausal women in relation to body size and composition. Breast Cancer Res Treat. 2009;115:171-9.

49. Zheng J, Haberland V, Baird D, Walker V, Haycock PC, Hurle MR, et al. Phenomewide Mendelian randomization mapping the influence of the plasma proteome on complex diseases. Nat Genet. 2020;52:1122-31.

50. Ritchie SC, Lambert SA, Arnold M, Teo SM, Lim S, Scepanovic P, et al. Integrative analysis of the plasma proteome and polygenic risk of cardiometabolic diseases. bioRxiv. 2021:2019.12.14.876474.

51. Brion MJ, Shakhbazov K, Visscher PM. Calculating statistical power in Mendelian randomization studies. Int J Epidemiol. 2013;42:1497-501.

52. Nikolaou CK, Hankey CR, Lean MEJ. Accuracy of on-line self-reported weights and heights by young adults. Eur J Public Health. 2017;27:898-903.

53. Burgess S, Davies NM, Thompson SG. Bias due to participant overlap in twosample Mendelian randomization. Genet Epidemiol. 2016;40:597-608.

54. Rosengren A, Smyth A, Rangarajan S, Ramasundarahettige C, Bangdiwala SI, AlHabib KF, et al. Socioeconomic status and risk of cardiovascular disease in 20 low-income, middle-income, and high-income countries: the Prospective Urban Rural Epidemiologic (PURE) study. Lancet Glob Health. 2019;7:e748-60.

55. Tillmann T, Vaucher J, Okbay A, Pikhart $H$, Peasey A, Kubinova R, et al. Education and coronary heart disease: mendelian randomisation study. BMJ. 2017;358:j3542.

\section{ACKNOWLEDGEMENTS}

Participants in the INTERVAL randomised controlled trial were recruited with the active collaboration of NHS Blood and Transplant England (www.nhsbt.nhs.uk), which has supported field work and other elements of the trial. DNA extraction and genotyping was co-funded by the National Institute for Health Research (NIHR), the NIHR BioResource (http://bioresource.nihr.ac.uk) and the NIHR [Cambridge Biomedical Research Centre at the Cambridge University Hospitals NHS Foundation Trust]*. The academic coordinating centre for INTERVAL was supported by core funding from: NIHR Blood and Transplant Research Unit in Donor Health and Genomics (NIHR BTRU2014-10024), UK Medical Research Council (MR/L003120/1), British Heart Foundation (SP/09/002; RG/13/13/30194; RG/18/13/33946) and the NIHR [Cambridge Biomedical Research Centre at the Cambridge University Hospitals NHS Foundation Trust]. A complete list of the investigators and contributors to the INTERVAL trial is provided in this reference [24]. The academic coordinating centre would like to thank blood donor centre staff and blood donors for participating in the INTERVAL trial. This work was supported by Health Data Research UK, which is funded by the UK Medical Research Council, Engineering and Physical Sciences Research Council, Economic and Social Research Council, Department of Health and Social Care (England), Chief Scientist Office of the Scottish Government Health and Social Care Directorates, Health and Social Care Research and Development Division (Welsh Government), Public Health Agency (Northern Ireland), British Heart Foundation and Wellcome. This work was also supported by the Wellcome Trust grant number 206194. *The views expressed are those of the authors and not necessarily those of the NHS, the NIHR or the Department of Health and Social Care.

\section{FUNDING}

This publication is the work of the authors who are guarantors for its contents. LG is funded by the University of Bristol alumni on the 4-year BHF Integrative Cardiovascular Science PhD programme. JAB is supported by the Elizabeth Blackwell Institute for Health Research, University of Bristol and the Wellcome Trust Institutional Strategic Support Fund (204813/Z/16/Z). DAH and LCC are funded by the Wellcome Trust (202802/Z/16/Z). KW is supported by the Wellcome Trust. GDS works in the Medical Research Council Integrative Epidemiology Unit at the University of Bristol, which is supported by the Medical Research Council (MC_UU_00011/1). NS is supported by the Wellcome Trust, British Heart Foundation and NIHR funding to the Cambridge Bioresource. JD is funded by the National Institute for Health Research (Senior Investigator Award). IH is supported by the EPSRC Prostanoid programme (EP/ M012530/1) and BHF (PG/16/3/31833). NJT is a Wellcome Trust Investigator (202802/ $\mathrm{Z} / 16 / \mathrm{Z}$ ), is the $\mathrm{PI}$ of the Avon Longitudinal Study of Parents and Children (MRC \& WT $102215 / 2 / 13 / 2$ ), is supported by the University of Bristol NIHR Biomedical Research 
Centre (BRC-1215-20011), the MRC Integrative Epidemiology Unit (MC_UU_12013/3) and works within the CRUK Integrative Cancer Epidemiology Programme (C18281/ A19169). The funders had no role in study design, data collection and analysis, decision to publish, or preparation of the paper. This research was funded in whole, or in part, by the Wellcome Trust (202802/Z/16/Z). For the purpose of Open Access, the author has applied a CC BY public copyright licence to any Author Accepted paper version arising from this submission.

\section{Declarations (Ethics):}

\section{COMPETING INTERESTS}

JD sits on the International Cardiovascular and Metabolic Advisory Board for Novartis (since 2010); the Steering Committee of UK Biobank (since 2011); the MRC International Advisory Group (ING) member, London (since 2013); the MRC High Throughput Science 'Omics Panel Member, London (since 2013); the Scientific Advisory Committee for Sanofi (since 2013); the International Cardiovascular and Metabolism Research and Development Portfolio Committee for Novartis; and the Astra Zeneca Genomics Advisory Board (2018).

\section{ADDITIONAL INFORMATION}

Supplementary information The online version contains supplementary material available at https://doi.org/10.1038/s41366-021-00896-1.
Correspondence and requests for materials should be addressed to L.J.G.

Reprints and permission information is available at http://www.nature.com/ reprints

Publisher's note Springer Nature remains neutral with regard to jurisdictional claims in published maps and institutional affiliations. adaptation, distribution and reproduction in any medium or format, as long as you give appropriate credit to the original author(s) and the source, provide a link to the Creative Commons license, and indicate if changes were made. The images or other third party material in this article are included in the article's Creative Commons license, unless indicated otherwise in a credit line to the material. If material is not included in the article's Creative Commons license and your intended use is not permitted by statutory regulation or exceeds the permitted use, you will need to obtain permission directly from the copyright holder. To view a copy of this license, visit http://creativecommons. org/licenses/by/4.0/.

(c) The Author(s) 2021 